\title{
SECOND SEASON PREDNISONE TREATMENT IN VERNAL CONJUNCTIVITIS WITH SPECIAL REFERENCE TO CORNEAL COMPLICATIONS*
}

\author{
BY \\ ELI NEUMANN \\ From the Department of Ophthalmology, Hadassah Medical School, Jerusalem
}

THIS work was started at the beginning of 1956 , when a research clinic for vernal conjunctivitis was set up in the Hadassah University Hospital, Jerusalem. Many intractable cases from throughout the country were sent for consultation and this accounts for the high percentage of severe cases in the series; it also partly explains why so many serious corneal complications were seen in a relatively short period.

\section{Classification of Patients}

Of some 400 cases of vernal conjunctivitis seen in the clinic since the beginning of 1956,100 were treated with prednisone.

Sufficient prednisone was not available for a longer series of cases, and those not so treated served as a control group.

Eighty-five of the cases receiving prednisone were classified as severe, and fifteen as moderate or mild. All the severe cases had some corneal involvement at one time or another. In most patients the disease was of the mixed variety, both palpebral and bulbar, but the presenting symptoms were due to the palpebral lesions in about two thirds of the cases.

The age and sex distribution of the hundred patients who received prednisone are given in Table I.

The duration of disease (Table I) varied from several weeks to 19 years (average 4 years).

TABLE I

AGE, SEX, AND DURATION OF DISEASE IN 100 PATIENTS

\begin{tabular}{|c|c|c|c|c|c|c|c|c|c|}
\hline \multicolumn{6}{|c|}{ Age (yrs) } & $1-5$ & $6-10$ & $11-15$ & Over 16 \\
\hline \multirow{3}{*}{$\begin{array}{l}\text { No. of } \\
\text { Patients }\end{array}$} & Total & .. & . & .. & .. & 9 & 30 & 32 & 29 \\
\hline & Male & .. & .. & .. & .. & 7 & 23 & 24 & 22 \\
\hline & Female & .. & .. & .. & .. & 2 & 7 & 8 & 7 \\
\hline \multicolumn{6}{|c|}{$\begin{array}{c}\text { Average Duration of Vernal } \\
\text { Conjunctivitis (yrs) } \ldots\end{array}$} & $2 \cdot 7$ & $3 \cdot 4$ & $3 \cdot 3$ & 5 \\
\hline
\end{tabular}

* Received for publication December 9, 1957. 


\section{Previous Treatment}

Most of the patients had already had various forms of treatment, including cortisone and hydrocortisone eye drops and ointment, local antibiotics, courses of desensitization injections, $\beta$-strontium irradiation, and surgery.

Patients were given prednisone instead of cortisone or hydrocortisone when these drugs were considered to be ineffective or not effective enough. It is worth while recording that some thirty patients gave a history of a good response to cortisone in the past, but with reduced benefit in the 1956 or 1957 season.

\section{Methods of Treatment}

Prednisone was given in the form of Metimyd eye drops $(0.5$ per cent. prednisone with 5 per cent. sulphacetamide: Schering) or Scherizolon (prednisone solution), diluted in saline to give a concentration of 0.25 per cent. The patients were asked to instil these drops three to five times daily initially, and less frequently as their condition improved.

Some patients were given additional Metimyd ointment for use before retiring at night.

25 very severe cases out of the 100 were also given courses of treatment with systemic prednisone. The initial dosage was $5 \mathrm{mg}$. three or four times daily, with a gradual decrease to a maintenance dose of $2.5 \mathrm{mg}$. three times daily for from 10 to 20 days. When necessary the course was repeated. We tried to prescribe this drug for short periods in the minimal effective dosage.

\section{Duration of Treatment}

The longest term of treatment was $1 \frac{1}{2}$ years and the shortest one month (average 6 months).

Sixty-five patients were started on prednisone therapy in 1956; fifteen of them have been under continuous treatment since then, receiving maintenance doses of Metimyd drops throughout the winter months. Thirty of the 65 patients were under treatment continuously during the summers of 1956 and 1957 and received no treatment during the winter months. In three cases in which treatment started in 1956, it was not necessary to continue treatment in 1957 because their clinical condition was satisfactory. The remaining seventeen patients did not reappear in the spring of 1957.

In 35 patients, prednisone treatment was commenced in the spring of 1957.

When prednisone therapy was started in 1956, thirty of the 65 patients were instructed to use Metimyd drops to the right eye and cortisone or hydrocortisone to the left eye. A week or 10 days later this order was reversed. However, as will be seen later, this procedure was soon stopped.

Cultures from the conjunctiva were taken from most cases and repeatedly from all cases suffering from corneal complications.

\section{Results}

The subjective criteria of effective treatment were taken to be diminution of irritation and of photophobia. The latter was usually one of the first 
symptoms to disappear. Objective criteria were diminution in the amount of secretion and changes in the diseased conjunctiva. The palpebral vegetations lost their redness, and became pale and flatter, and in some cases completely disappeared within a few weeks. In bulbar cases the conjunctiva became white, and the limbal proliferations became flatter and in some cases eventually disappeared.

The Horner-Trantas spots usually disappeared in a few days and their continuance was considered an indication for increasing the dose of prednisone.

Of the corneal complications, the disappearance of superficial keratitis with its symptoms of photophobia and blepharospasm was a definite criterion as to the effect of therapy. The improvement in the corneal condition was sometimes noted within the first few days of treatment, even in patients who had been seriously disabled for weeks or months.

Most of these results could be obtained with local prednisone, but the more severe symptoms such as large limbal proliferations and torpid corneal ulcers could be more quickly controlled with systemic prednisone.

After intermission or cessation of treatment or when maintenance doses were inadequate, some relapses occurred. Resumption of or increase in dosage, however, soon brought the symptoms under control again.

Judging by these criteria, prednisone was superior to all other forms of therapy. Its superiority to cortisone and hydrocortisone was clearly brought out by the experiment in which thirty patients received prednisone to one eye and cortisone or hydrocortisone to the other eye.

In order to assess the relative value of cortisone and hydrocortisone as compared with prednisone in the management of vernal conjunctivitis, the results in the 100 cases treated with cortisone or hydrocortisone are compared with those in the 100 cases treated with prednisone (Table II).

TABLE II

COMPARATIVE EFFECTS OF CORTISONE, HYDROCORTISONE, AND PREDNISONE

\begin{tabular}{|c|c|c|c|c|c|}
\hline Type of Response & & Good & Medium & Little or None & Total \\
\hline Cortisone and Hydrocortisone & .. & 8 & 36 & 56 & 100 \\
\hline Prednisone & .. & 100 & 0 & 0 & 100 \\
\hline
\end{tabular}

Corneal Complications.-Of the 100 patients treated with prednisone five developed corneal ulcerations while under maintenance doses of Metimyd. The ulceration occurred from three to eight months after Metimyd was started. Fuller data relating to these five patients are given in Table III (opposite). 
TABLE III

CLINICAL DATA RELATING TO CORNEAL ULCERATION IN FIVE PATIENTS

\begin{tabular}{r|c|c|c|c|c|c}
\hline $\begin{array}{c}\text { Case } \\
\text { No. }\end{array}$ & $\begin{array}{c}\text { Age } \\
\text { (yrs) }\end{array}$ & Sex & $\begin{array}{c}\text { Duration of Vernal } \\
\text { Conjunctivitis (yrs) }\end{array}$ & $\begin{array}{c}\text { Length of Prednisone } \\
\text { Treatment (mths) }\end{array}$ & Site of Ulcer & Side \\
\hline 5 & 5 & Male & 4 & $3 \cdot 5$ & $\begin{array}{c}\text { Upper part of } \\
\text { cornea }\end{array}$ & Right \\
\hline 49 & 8 & Male & 3 & 8 & $\begin{array}{c}\text { Upper part of } \\
\text { cornea }\end{array}$ & Right \\
\hline 73 & 5 & Female & 4 & $\begin{array}{c}\text { Upper para- } \\
\text { central }\end{array}$ & Left \\
\hline 178 & 5 & Male & 3 & $\begin{array}{c}\text { Upper part of } \\
\text { cornea }\end{array}$ & Right \\
\hline 205 & 4 & Male & 3 & $\begin{array}{c}\text { Upper para- } \\
\text { central }\end{array}$ & Both \\
\hline
\end{tabular}

- All the above cases were under steroid therapy (cortisone and later prednisone) almost from the start of the disease. This serious complication occurred in small children only, and the age at onset was remarkably low. Reference to Table I shows that four out of nine in the 1 to 5-year-old age group developed corneal ulceration, whereas only one out of the remaining 91 patients of all other ages was affected in this way.

All the ulcers had a torpid course and did not respond to therapy unless prednisone was given orally, when healing progressed satisfactorily and was complete within a week or two.

In all five cases a nebula or macula remained at the site of the ulcer, and relapses at the same site or around it were common. Although there was only one bilateral case, the other cases showed a tendency to erosions in the second eye. The visual acuity was not appreciably affected, but there were 3 to $4 \mathrm{D}$ astigmatism in two cases. All five children had either the palpebral or the mixed form of the disease. There was no trachoma in any of the five cases.

One patient only (Case 49) received $\beta$-strontium irradiations; the dosage, however, was small (3000 rep.) and the source was applied directly to the everted upper eyelid only. It is improbable that in this case these irradiations were the cause of the ulcer.

When the ulcerations appeared the vegetations on the conjunctiva of the upper eyelids were already so small and scarce that surgical intervention as advocated by Beigelman (1950) was not considered. In four cases the conjunctival cultures showed Staphylococcus albus, which was particularly sensitive to chloramphenicol; one culture was sterile. Treatment with chloramphenicol without systemic prednisone did not affect the ulceration. 


\section{Discussion}

Of 48 cases treated with prednisone during two seasons, only three were considered to be cured, although in the seventeen patients treated by prednisone in the summer of 1956 who did not return in 1957 some cures may be assumed. The general impression is that the rate of cure in the group treated by prednisone was not greater than that to be naturally expected. Prednisone is probably the most effective drug at the present time in the management of vernal conjunctivitis, but there is no proof that it cures the disease.

The relation between steroid therapy and the apparent increase in frequency of corneal ulceration in vernal conjunctivitis requires further consideration. In our series five cases of corneal ulcer occurred while the patient was receiving prednisone. This may be unrelated to the therapy, and due rather to the fact that the prednisone-treated cases were nearly all severe. Prednisone by mouth quickly healed the corneal ulceration. Corneal ulceration also developed in a number of cases in which steroids other than prednisone were given, its incidence being proportional to the severity of the primary condition.

It is well known that corneal ulceration occurred as a complication of vernal conjunctivitis before steroid therapy was introduced. However, we were concerned with the possibility that prednisone might be more liable than cortisone to cause corneal ulceration. Of 300 cases of vernal conjunctio vitis treated by cortisone, 2.66 per cent. developed corneal ulceration. The difference between this figure and the 5 per cent. found in the prednisones treated group may be explained by the fact that the latter comprised more severe cases.

There was no evidence that infection played a part in the five cases of corneal ulceration described-a role which might conceivably have been attributed to the influence of steroids in lowering local resistance to infection. It is now our practice to give only systemic prednisone in cases of vernal conjunctivitis in which corneal ulceration has already appeared, though this may well be an unnecessary precaution.

\section{Summary}

(1) A hundred patients with vernal conjunctivitis received prednisone treatment, the majority during two consecutive years.

(2) The clinical data of these cases is supplied.

(3) During the same period 300 other cases of vernal conjunctivitis were treated without prednisone. These cases were used as controls.

(4) Prednisone was found to be superior to all other steroids in the management of vernal conjunctivitis.

(5) There is no evidence that it is effective in curing the disease. 
(6) There is no conclusive evidence that it increases the liability to corneal ulceration, but, as a precautionary measure, the drug was given by mouth only in such cases. Further experience may show this precaution to be unnecessary.

The author wishes to express his indebtedness to Professor I. C. Michaelson, for his helpful advice and guidance throughout this work, which was supported by the Max and Rebecca Schrire Medical Research Fellowship. Messrs. Schering Corporation supplied the prednisone preparations used in the investigations.

\section{REFERENCE}

Beigelman, M. N. (1950). “Vernal Conjunctivitis”. University of Southern California Press, Los Angeles, U.S.A. 